\title{
Supporting Information: Disentangling Sum- Frequency Generation Spectra of the Water Bending Mode at Charged Aqueous Interfaces
}

Takakazu Seki, ${ }^{1, \S}$ Chun-Chieh Yu, ${ }^{1,}$ Kuo-Yang Chiang, ${ }^{1}$ Junjun Tan, ${ }^{2}$ Shumei Sun, ${ }^{3}$ Shuji Ye, ${ }^{2}$ Mischa Bonn, ${ }^{1}$ and Yuki Nagata ${ }^{1, *}$

1. Max Planck Institute for Polymer Research, Ackermannweg 10, 55128 Mainz, Germany

2. Hefei National Laboratory for Physical Sciences at the Microscale, and Department of Chemical Physics, University of Science and Technology of China, 230026 Hefei, China.

3. Department of Physics and Applied Optics Beijing Area Major Laboratory, Beijing Normal University, Beijing 100875, China

*Correspondence: nagata@mpip-mainz.mpg.de

$\S:$ These authors contributed equally.

\section{HD-SFG Measurements (Nagata/Bonn group)}

The HD-SFG measurements were performed on a collinear beam geometry using a Ti: Sapphire regenerative amplifier (Spitfire Ace, Spectra-Physics, centered at $800 \mathrm{~nm}, \sim 40 \mathrm{fs}$ pulse duration, $5 \mathrm{~mJ}$ pulse energy, $1 \mathrm{kHz}$ repetition rate). A part of the output was used to generate a broadband infrared (IR) pulse in an optical parametric amplifier (Light Conversion TOPAS-C) with a silver gallium disulfide $\left(\mathrm{AgGaS}_{2}\right)$ crystal. The other part of the output was directed through a pulse shaper consisting of a grating-cylindrical mirror system to generate a narrowband visible pulse with a bandwidth of $\sim 10 \mathrm{~cm}^{-1}$. The IR and 
visible beam were firstly focused into a $20 \mu \mathrm{m}$-thick y-cut quartz plate as the local oscillator (LO). Then these beams were collinearly passed through an $8 \mathrm{~mm} \mathrm{CaF}_{2}$ plate for the phase modulation and focused onto the sample surface at angles of incidence of $45^{\circ}$. The SFG signal from the sample is interfered with the SFG signal from the LO, generating the SFG interferogram. The SFG interferogram was dispersed in a spectrometer (Andor Technology, Shamrock 193i) and detected by an EMCCD camera (Andor Technology, Newton). The

complex spectra of second-order nonlinear susceptibility $\left(\chi_{\mathrm{eff}}^{(2)}\right)$ were obtained via the Fourier analysis of the interferogram and normalization by a z-cut quartz crystal. The measurements were performed with $s s p$ (denoting $s-, s-$, and $p$-polarized SFG, visible, and IR beams, respectively) polarization combination.

\section{HD-SHG Measurements (Nagata/Bonn group)}

In the HD-SHG experiments, a pulsed Yb:KGW (ytterbium-doped potassium gadolinium tungstate) laser system (Pharos, Light Conversion Ltd) was used, generating pulses with a wavelength of $\sim 1030 \mathrm{~nm}$, a pulse duration of roughly $210 \mathrm{fs}$, a repetition rate of $1 \mathrm{MHz}$ and a pulse energy of $15 \mu \mathrm{J}$. The pulse energy was set to $300 \mathrm{~nJ}$. The fundamental beam, after passing through a $y$-cut quartz acting as a LO and fused silica plates for phase modulation, was focused onto the sample surface. All the measurements were performed with $s$-in/ $p$-out polarization combinations. The incident angle of the incoming beam was set to $45^{\circ}$ relative to the surface normal. The generated SHG beam was dispersed in a spectrograph (Princeton Instruments, Acton SP2300) and detected by a EMCCD (Andor Technology, iXon). The complex spectra of second-order nonlinear susceptibility $\left(\chi_{\text {eff }}^{(2)}\right)$ were obtained via the operation of Fourier transformation, filtering the time-domain signal, and inverse Fourier transformation. The obtained spectra of the sample were normalization by the spectra for the $z$-cut quartz crystal. 


\section{Sample Preparation (Nagata/Bonn group)}

For the lipid solution, we dissolved DPPG (sodium salt) and DPTAP (chloride salt) purchased from Avanti Polar Lipids in a mixture of 90\% chloroform (Fischer Scientific, stabilized with amylene, $>99 \%$ ) and 10\% methanol (VWR Chemicals, 99.8\%) at a concentration of $4.3 \times 10^{-4} \mathrm{~mol} / \mathrm{L}$. Sodium chloride (Sigma Aldrich, $>99.5 \%$ ) was baked in an oven for 8 hours at $650{ }^{\circ} \mathrm{C}$. We used $\mathrm{D}_{2} \mathrm{O}(>99.9 \%)$, which was purchased from SigmaAldrich. We used $\mathrm{H}_{2} \mathrm{O}$, which was obtained from a MilliQ machine (the resistance of 18.2 $\mathrm{M} \Omega \mathrm{cm}$ ). We dissolved salt into the $\mathrm{H}_{2} \mathrm{O}$ and $\mathrm{D}_{2} \mathrm{O}$ and made the salt solutions.

The $20 \mathrm{~mL}$ salt solutions were poured into a Teflon trough with a diameter of $\sim 8.0$ cm. Subsequently, we added the lipid solution onto the $\mathrm{H}_{2} \mathrm{O}$ and $\mathrm{D}_{2} \mathrm{O}$ solutions using a click syringe. The surface pressure of the DPTAP and DPPG monolayers were measured with a commercial surface tension meter (KBN 315 Sensor Head, Kibron, Inc., Helsinki, Finland) and were determined to be $\sim 44 \pm 3 \mathrm{mN} / \mathrm{m}$ and $\sim 19 \pm 3 \mathrm{mN} / \mathrm{m}$, respectively. The surface area per lipid was estimated to be $44 \AA^{2}$ and $52 \AA^{2}$ for DPTAP and DPPG, respectively ${ }^{1,2}$. The prepared samples were equilibrated over at least $40 \mathrm{~min}$. For both HDSFG and HD-SHG measurements, the trough was rotated with a speed of $\sim 1.0 \mathrm{~cm}$ per sec with respect to the laser irradiation spot (Fig. S1). The rotating trough was needed to avoid the heat accumulation, which may cause the Marangoni flow and thus alter the lipid conformation. $^{3}$

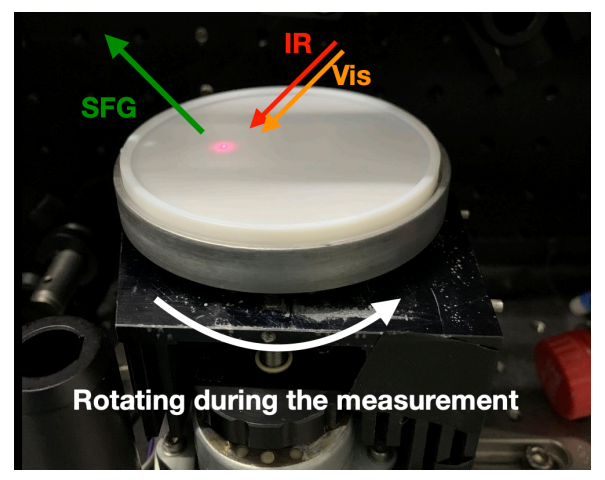

Figure S1. Photograph of the rotating trough and beam position on the sample. 
We measured the fluctuation of the height due to the rotating stage via the Keyence height displacement sensor (LK-G82). The standard deviation of the height was $1.7 \mu \mathrm{m}$. Since the non-collinear HD-SFG measurement requires the accuracy of $1 \mu \mathrm{m},{ }^{4}$ the combination of the rotating trough with the collinear HD-SFG setup provides the unique solution to measure the water-lipid interface by avoiding heat accumulation.

\section{Homodyne-SFG Measurements with Picosecond Laser Setup (Ye group)}

The homodyne-SFG measurements were carried out using a commercial EKSPLA SFG system with a repetition rate of $10 \mathrm{~Hz}$ and a pulse width of 30 picoseconds. The SFG spectra were collected by overlapping a visible and a tunable IR beam on the sample surface using a co-propagating configuration. The visible beam $\left(\omega_{\mathrm{V} \text { is }}\right)$ was set at a fixed wavelength of $532.1 \mathrm{~nm}$ while the IR beam $\left(\omega_{\mathrm{IR}}\right)$ can be tuned from 1500 to $1800 \mathrm{~cm}^{-1}$. The angles of incidence are $63^{\circ}$ and $53^{\circ}$ for the visible and IR laser beams, respectively. The ssp SFG spectra from the water-lipid interface were collected with the spectral resolution of $5 \mathrm{~cm}^{-1}$. Each spectral data point in the SFG spectra was averaged 100 shots and normalized by the intensities of the input IR and visible beams before collecting the next data point. The experiments were carried out at room temperature $\left(25^{\circ} \mathrm{C}\right)$.

\section{Sample Preparation (Ye group)}

Lipid DPTAP was purchased from Avanti Polar Lipids (Alabaster, AL). Sodium chloride $(>99.8 \%)$ and $\mathrm{D}_{2} \mathrm{O}(>99.9 \%)$ were purchased from Aldrich. DPTAP was dissolved in chloroform (purchased from Sinopharm Chemical Reagent Co., Ltd.) at a concentration of $1.0 \mathrm{mg} / \mathrm{mL}$. The salt was baked at around $500^{\circ} \mathrm{C}$ for more than 6 hours to remove the organic impurities. The ultrapure water (the resistance of $18.2 \mathrm{M} \Omega \mathrm{cm}$ ) was produced by Millipore system (Millipore, Bedford, MA).

The $\sim 25 \mathrm{~mL}$ salt solutions were poured into a Teflon trough with a diameter of $\sim 8.0$ cm. Subsequently, we added the DPTAP lipid solution onto the $\mathrm{H}_{2} \mathrm{O}$ and $\mathrm{D}_{2} \mathrm{O}$ solutions 
using a click syringe to get a target surface pressure of $\sim 44 \mathrm{mN} / \mathrm{m}$, which was measured with a commercial surface tension meter (BZY-A, Shanghai Fangrui Instrument, Inc., Shanghai, China). It was then given $\sim 30$ minutes to allow solvent evaporation and to reach surface pressure equilibrium. The trough was rotated with a speed of $\sim 18$ degree/sec to avoid heat accumulation due to the laser irradiation spot (similar to Fig. S1).

\section{Frequency variation of the $C=O$ Stretch Mode}

In Figure $\mathrm{S} 2$, we compare the characteristic frequency of the $\mathrm{C}=\mathrm{O}$ stretch mode in the $\left|\chi_{\text {eff }}^{(2)}\right|^{2}$ spectra measured by the Nagata/Bonn group (Current work and Ref. 5) and that reported in Ref. 6 for the $\mathrm{D}_{2} \mathrm{O}$-DPTAP interface. The constructed $\left|\chi_{\mathrm{eff}}^{(2)}\right|^{2}$ data (red line in Fig. S2) from the HD-SFG measurement is consistent with the homodyne-detected $\left|\chi_{\text {eff }}^{(2)}\right|^{2}$ data (orange line in Fig. S2), though the geometries of the setups and the lipid density are slightly different. ${ }^{7}$ The $\mathrm{C}=\mathrm{O}$ stretch mode frequency is $\sim 1730 \mathrm{~cm}^{-1}$ for both data. On the other hand, these data differ from data reported by the Tahara group (green line in Fig. S2). The $\mathrm{C}=\mathrm{O}$ stretch mode frequency is $\sim 1740 \mathrm{~cm}^{-1}$ for the data obtained by Tahara group. Because the lower surface coverage results in the lower amplitude and blue-shift of the $\mathrm{C}=\mathrm{O}$ stretch peak, ${ }^{8}$ it is very likely that the coverage of the lipid for the laser spot is drastically reduced, and thus the net surface charge in the probed region is significantly reduced in Ref. 6. To check the data reproducibility, we performed independent SFG measurements in the Ye group with the picosecond laser SFG system. The results are given with purple dots in Fig. S2. The data obtained from the Ye group also shows the characteristic frequency of $\sim 1730 \mathrm{~cm}^{-1}$. We used the rotational stage of the sample holder, avoiding the heat accumulation due to the laser radiation and thus allowing us to measure the SFG signal at the lipid interfaces without distortion of the lipid monolayer while the Tahara group did not rotate the samples during the measurements. Apparently, the different SFG response of the $\mathrm{C}=\mathrm{O}$ stretch mode in Ref. 6 manifests that the formations of the lipid monolayer are significantly different. 


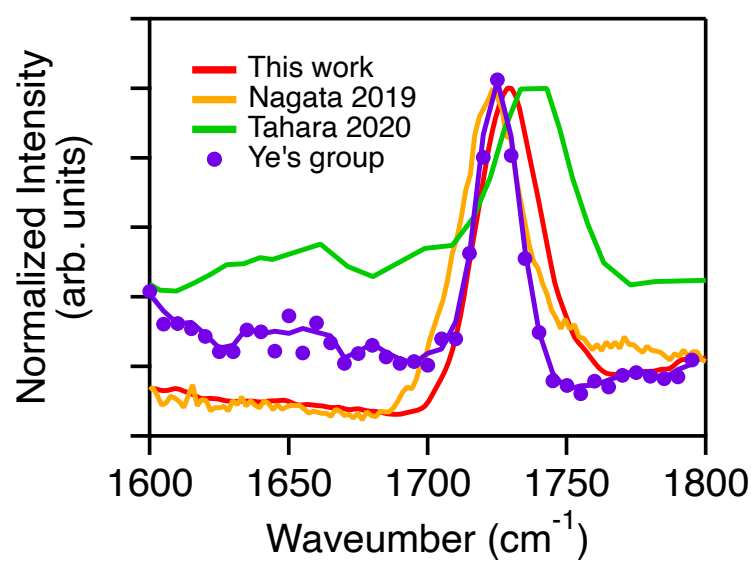

Figure S2. The $\left|\chi_{\text {eff }}^{(2)}\right|^{2}$ spectra at the $\mathrm{D}_{2} \mathrm{O}$-DPTAP interfaces with salt concentrations of 1 $\mathrm{M}$ and $0.5 \mathrm{M}$ (Tahara 2020). All the data sets are normalized to the intensity of the $\mathrm{C}=\mathrm{O}$ stretch mode signal. The solid purple line is to guide the eye for Ye's group data. The data labeled as "Nagata 2019" and "Tahara 2020" are reproduced from Refs. 6 and 7, respectively.

\section{Fitting Procedure for HD-SFG Spectra of Water Bending Mode}

We performed the spectral fitting for the HD-SFG spectra $\left(\operatorname{Im}\left(\chi_{\text {eff }}^{(2)}\right)\right.$ and $\left.\operatorname{Re}\left(\chi_{\text {eff }}^{(2)}\right)\right)$ via

$$
\chi_{\mathrm{eff}}^{(2)}(\omega)=A_{\mathrm{NR}} e^{i \phi_{\mathrm{NR}}}+\sum_{i=1,2} \chi_{\mathrm{C}=0, i}^{(2)}(\omega)+\chi_{\mathrm{bend}}^{(2)}(\omega)+\chi_{\mathrm{bend}}^{(3)}(\omega) \Phi(\sigma, c) \frac{\kappa(c)}{\kappa(c)-i \Delta k_{z}}
$$

for the $\mathrm{H}_{2} \mathrm{O}$-lipid samples and via

$$
\chi_{\mathrm{eff}}^{(2)}(\omega)=A_{\mathrm{NR}} e^{i \phi_{\mathrm{NR}}}+\sum_{i=1,2} \chi_{\mathrm{C}=0, i}^{(2)}(\omega)
$$


for the $\mathrm{D}_{2} \mathrm{O}$ - lipid samples. Here, $A_{N R}\left(\phi_{N R}\right)$, and $\chi_{\mathrm{C}=\mathrm{O}, i}^{(2)}(\omega)$ are the amplitude (phase) of the non-resonant contribution and the second-order susceptibility of the $\mathrm{C}=\mathrm{O}$ stretch mode of carbonyl group of the lipid, respectively. $\chi_{\text {bend }}^{(2)}(\omega)$ and $\chi_{\text {bend }}^{(3)}(\omega)$ represent the secondorder and third-order susceptibilities of the H-O-H bending mode. $\sigma$ and $c$ represent surface charge density and ion concentration. $\Phi(\sigma, c)$ and $\kappa(c)$ denote the surface potential and the inverse of the Debye length, respectively. $\Delta k_{z}$ denotes the mismatch of the wavevectors along $z$ axis in the reflected SFG configuration.

In the Gouy-Chapman model, the surface potential $\Phi(\sigma, c)$ is given by ${ }^{9}$

$$
\Phi_{0}(\sigma, c)=\frac{2 k_{B} T}{e_{c}} \sinh ^{-1}\left(\frac{\sigma}{\sqrt{8000 k_{B} T N_{A} \varepsilon_{0} \varepsilon_{r} c}}\right)=0.052 \cdot \sinh ^{-1}\left(\frac{\sigma}{0.117 \sqrt{c}}\right)
$$

The inverse of the Debye length $\kappa(c)$ is given by ${ }^{9}$

$$
\kappa(c)=\sqrt{\frac{2000 e_{c}^{2} N_{A} c}{\varepsilon_{0} \varepsilon_{r} k_{B} T}}=\frac{\sqrt{c}}{0.304}
$$

where $k_{B}, T, e_{c}, N_{A}, \varepsilon_{0}$, and $\varepsilon_{r}$ represent the Boltzmann constant, temperature, the elementary charge, the Avogadro's number, the vacuum permittivity, and the relative permittivity of water, respectively. The surface charge density was assumed to be +0.35 $\mathrm{C} / \mathrm{m}^{2}$ and $-0.30 \mathrm{C} / \mathrm{m}^{2}$ for DPTAP and DPPG, respectively, based on the surface area per lipid. The mismatch of the wave-vectors, $\Delta k_{z}$, was calculated via;

$$
\Delta k_{z}=k_{\mathrm{SFG}, Z}+k_{\mathrm{vis}, z}+k_{\mathrm{IR}, z},
$$

and

$$
k_{i, z}=\frac{2 \pi}{\lambda} \sqrt{n_{i}^{2}-\sin ^{2} \theta_{i}}(i=\mathrm{SFG}, \text { vis, or IR })
$$


where $k_{i, z}, \lambda, n_{i}$, and $\theta_{i}$ indicate wave-vector at z-plane for the beam $(i)$, wavelength, refractive index of water corresponding to the beam $(i)$, and beam angle with respect to the surface normal, respectively. We obtained $\Delta k_{z}=49\left(\mathrm{~nm}^{-1}\right)$.

For $\mathrm{C}=\mathrm{O}$ stretch mode peaks $\left(\chi_{\mathrm{C}=0,1}^{(2)}(\omega)\right.$ and $\left.\chi_{\mathrm{C}=0,2}^{(2)}(\omega)\right)$, we assumed a Lorentz shape

$$
\chi_{\mathrm{C}=0, \mathrm{i}}^{(2)}(\omega)=\frac{A_{\mathrm{C}=0, \mathrm{i}}}{\omega-\omega_{\mathrm{C}=0, \mathrm{i}}+i \Gamma_{\mathrm{C}=0, \mathrm{i}}},
$$

where $A_{\mathrm{C}=\mathrm{O}, i}, \omega_{\mathrm{C}=\mathrm{O}, i}$, and $\Gamma_{\mathrm{C}=\mathrm{O}, i}$ denote the amplitude, characteristic frequency and a half width at half maxima of the $i$ th $\mathrm{C}=\mathrm{O}$ stretch mode, respectively $(i=1,2)$.

For the water's bending mode contribution $\left(\chi_{\text {bend }}^{(2)}(\omega)\right.$ and $\left.\chi_{\text {bend }}^{(3)}(\omega)\right)$, we assumed the Voigt profile;

$$
\begin{aligned}
& \chi_{\text {bend }}(\omega)=\frac{A_{\text {bend }}}{\omega-\omega_{\text {bend }}+i \Gamma_{\text {bend,hom }}} \otimes \frac{1}{\sqrt{2 \pi} \Gamma_{\text {bend,inh }}} \exp \left(-\frac{\omega^{2}}{2 \Gamma_{\text {bend,inh }}^{2}}\right) \\
& =\frac{1}{\sqrt{2 \pi} \Gamma_{\text {bend,inh }}} \int_{0}^{\infty} \frac{A_{\text {bend }}}{\omega_{\text {bend }}-\omega^{\prime}+i \Gamma_{\text {bend,hom }}} \exp \left(-\frac{\left(\omega-\omega^{\prime}\right)^{2}}{2 \Gamma_{\text {bend,inh }}^{2}}\right) d \omega^{\prime},
\end{aligned}
$$

where $A_{\text {bend }}, \omega_{\text {bend }}$, and $\Gamma_{\text {bend,hom denote the amplitude, characteristic frequency and }}$ homogeneous broadening, and $\Gamma_{\text {bend,inh }}$ accounts for inhomogeneous broadening. To reduce the degrees of freedom on the fits, we assumed that $2 \sqrt{2 \ln 2} \Gamma_{\text {bend,inh }}$ (full-width at half maxima of the gaussian distribution) and $\omega_{\text {bend }}$ for $\chi_{\text {bend }}^{(3)}(\omega)$ were set to $35 \mathrm{~cm}^{-1}$ and $1650 \mathrm{~cm}^{-1}$, respectively. We made constraints on $\Gamma_{\text {bend,hom }}$ and $\Gamma_{\text {bend,inh }}$ for the $\chi_{\text {bend }}^{(2)}(\omega)$ contribution and $A_{\text {bend }}, \omega_{\text {bend }}, \Gamma_{\text {bend,hom }}$, and $\Gamma_{\text {bend,inh }}$ for the $\chi_{\text {bend }}^{(3)}(\omega)$ contribution to have the same values between the DPTAP and DPPG samples. The best fits were obtained with the $\Gamma_{\text {bend,hom }}$ of $15 \mathrm{~cm}^{-1}$. 
Note that the fitting has been done with a Lorentzian lineshape in our previous study $^{5}$, whereas the $\operatorname{Im}\left(\chi_{\text {eff }}^{(2)}(\omega)\right)$ spectra in the HD-SFG measurement shows that the bending mode contribution is not Lorentzian shape, rather close to the Gaussian shape. Thus, we decided to use the Voigt profile for fit the data.

To capture the $\mathrm{H}-\mathrm{O}-\mathrm{H}$ bending contribution, we performed a two-step fitting procedure for the $\operatorname{Im}\left(\chi_{\text {eff }}^{(2)}(\omega)\right)$ spectra of the $\mathrm{H}_{2} \mathrm{O}$-lipid and $\mathrm{D}_{2} \mathrm{O}$-lipid interfaces via equations $\mathrm{S} 1$ and $\mathrm{S} 2$, respectively. With the shared information of $\omega_{\mathrm{C}=0, i}$, and $\Gamma_{\mathrm{C}=0, i}$, we performed global fits for the imaginary part SFG spectra with various salt concentrations in a similar manner to our previous work. ${ }^{5}$ After extracting that the vibrationally-resonant responses from the imaginary part, we further performed fits for the $\operatorname{Re}\left(\chi_{\text {eff }}^{(2)}(\omega)\right)$ spectra to describe the non-resonant contributions. Through the fits, we obtained $\chi_{\text {bend }}^{(2)}(\omega)=$ $\frac{1}{\sqrt{2 \pi} 15} \int_{0}^{\infty} \frac{0.021}{1672-\omega^{\prime}+15 \mathrm{e}} \exp \left(-\frac{\left(\omega-\omega^{\prime}\right)^{2}}{2 \times 15^{2}}\right) d \omega^{\prime}$ for the water-DPTAP interface, $\chi_{\text {bend }}^{(2)}(\omega)=$ $\frac{1}{\sqrt{2 \pi} 15} \int_{0}^{\infty} \frac{-0.13}{1652-\omega^{\prime}+15 \mathrm{i}} \exp \left(-\frac{\left(\omega-\omega^{\prime}\right)^{2}}{2 \times 15^{2}}\right) d \omega^{\prime}$ for the water-DPPG interface, and $\chi_{\text {bend }}^{(3)}(\omega)=$ $\frac{1}{\sqrt{2 \pi} 15} \int_{0}^{\infty} \frac{-0.54}{1650-\omega^{\prime}+15 \mathrm{i}} \exp \left(-\frac{\left(\omega-\omega^{\prime}\right)^{2}}{2 \times 15^{2}}\right) d \omega^{\prime}$. The fitting curves are plotted in Figures S1 and S2, and the results are summarized in Tables S1 and S2.

The obtained sign of the $\operatorname{Im}\left(\chi_{\text {bend }}^{(2)}(\omega)\right)$ and $\operatorname{Im}\left(\chi_{\text {bend }}^{(3)}(\omega) \Phi(c)\right)$ spectra are consistent with our previous study. ${ }^{5}$ However, critical comparison of the current data set obtained from the HD-SFG with the previous data set obtained from the homodynedetected SFG indicates that some fit parameters are not accurately obtained because of the uncertainty of the fit. First, a broad peak feature at $\sim 1800 \mathrm{~cm}^{-1}$ assumed based on Ref. 8 is not present in the current HD-SFG data. This misleadingly assumed $1800 \mathrm{~cm}^{-1}$ peak affects the fit of the non-resonant background. Second, the characteristic frequency of the obtained $\chi_{\text {bend }}^{(2)}(\omega)$ peak at the water-DPTAP interface was $\sim 1645 \mathrm{~cm}^{-1}$ in our previous study, while the frequency obtained in this study was $\sim 1670 \mathrm{~cm}^{-1}$. These illustrate that, for rigorous discussion of the bending mode frequency, the HD-SFG technique is highly required. 


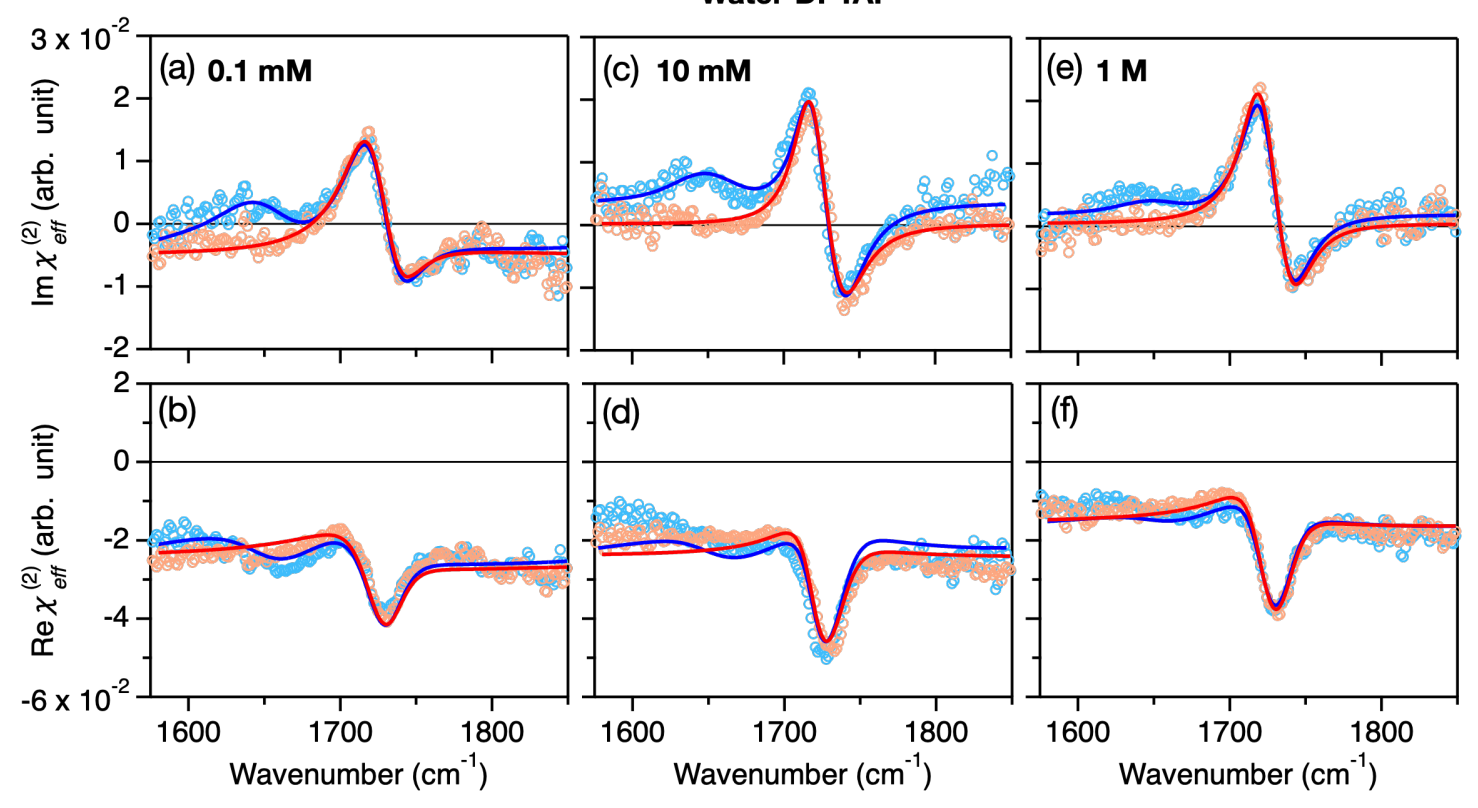

Figure S3. (a-f) HD-SFG spectra at the $\mathrm{H}_{2} \mathrm{O}$-DPTAP interface (blue) and at the $\mathrm{D}_{2} \mathrm{O}$ DPTAP interface (red) with various $\mathrm{NaCl}$ concentrations. The solids lines represent the fits.
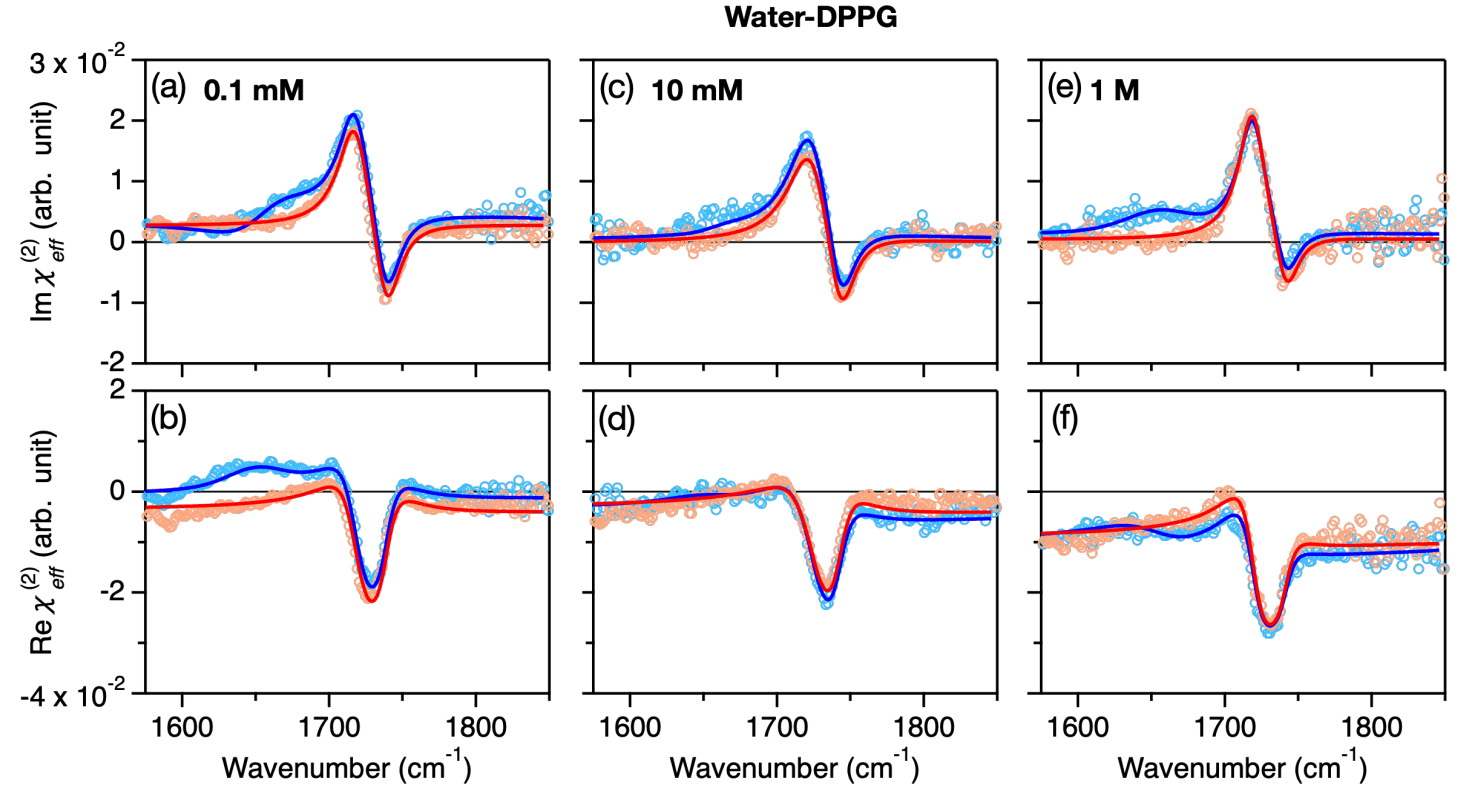

Figure S4. (a-f) HD-SFG spectra at the $\mathrm{H}_{2} \mathrm{O}-\mathrm{DPPG}$ interface (blue) and at the $\mathrm{D}_{2} \mathrm{O}-\mathrm{DPPG}$ interface (red) with various $\mathrm{NaCl}$ concentrations. The solids lines represent the fits. 
Table S1. The fitting parameters of the complex SFG spectra at water-DPTAP interfaces. The error was obtained from the fits.

\begin{tabular}{|c|c|c|c|c|c|c|}
\hline DPTAP & $\mathrm{H}_{2} \mathrm{O}$ & $\mathrm{D}_{2} \mathrm{O}$ & \multicolumn{2}{c|}{$\mathrm{H}_{2} \mathrm{O}$} & $\mathrm{D}_{2} \mathrm{O}$ & \multicolumn{2}{c|}{$\mathrm{H}_{2} \mathrm{O}$} & $\mathrm{D}_{2} \mathrm{O}$ \\
\hline $\mathrm{NaCl}$ & \multicolumn{2}{|c|}{$1 \mathrm{M}$} & \multicolumn{2}{c|}{$10 \mathrm{mM}$} & \multicolumn{2}{c|}{$0.1 \mathrm{mM}$} \\
\hline$A_{\mathrm{NR}}$ & $0.016 \pm 0.001$ & $0.016 \pm 0.001$ & $0.023 \pm 0.001$ & $0.024 \pm 0.001$ & $0.024 \pm 0.001$ & $0.026 \pm 0.001$ \\
\hline$\phi_{\mathrm{NR}}(\mathrm{rad})$ & $3.03 \pm 0.01$ & $3.12 \pm 0.01$ & $2.98 \pm 0.03$ & $3.13 \pm 0.04$ & $3.30 \pm 0.04$ & $3.34 \pm 0.03$ \\
\hline$A_{\mathrm{C}=\mathrm{O}, 1}$ & $-0.45 \pm 0.08$ & $-0.51 \pm 0.08$ & $-0.42 \pm 0.05$ & $-0.45 \pm 0.05$ & $-0.71 \pm 0.15$ & $-0.72 \pm 0.14$ \\
\hline$\omega_{\mathrm{C}=\mathrm{O}, 1}\left(\mathrm{~cm}^{-1}\right)$ & \multicolumn{2}{|c|}{$1720.7 \pm 1.0$} & \multicolumn{2}{|c|}{$1718.5 \pm 0.7$} & \multicolumn{2}{c|}{$1721.3 \pm 2.5$} \\
\hline$\Gamma_{\mathrm{C}=\mathrm{O}, 1}\left(\mathrm{~cm}^{-1}\right)$ & \multicolumn{2}{|c|}{$16.4 \pm 1.0$} & \multicolumn{2}{|c|}{$14.7 \pm 0.9$} & \multicolumn{2}{c|}{$23.2 \pm 1.5$} \\
\hline$A_{\mathrm{C}=\mathrm{O}, 2}$ & $0.38 \pm 0.08$ & $0.39 \pm 0.08$ & $0.48 \pm 0.05$ & $0.41 \pm 0.05$ & $0.48 \pm 0.14$ & $0.45 \pm 0.13$ \\
\hline$\omega_{\mathrm{C}=\mathrm{O}, 2}\left(\mathrm{~cm}^{-1}\right)$ & \multicolumn{2}{|c|}{$1738.5 \pm 1.6$} & \multicolumn{2}{|c|}{$1736.4 \pm 1.2$} & \multicolumn{2}{c|}{$1736.0 \pm 2.2$} \\
\hline$\Gamma_{\mathrm{C}=\mathrm{O}, 2}\left(\mathrm{~cm}^{-1}\right)$ & \multicolumn{2}{|c|}{$17.8 \pm 1.3$} & $19.3 \pm 1.1$ & \multicolumn{2}{c|}{$20.3 \pm 2.2$} \\
\hline$A_{\text {bend }}$ for $\chi_{\text {bend }}^{(2)}$ & $0.021 \pm 0.007$ & $\mathrm{NA}$ & $0.021 \pm 0.007$ & $\mathrm{NA}$ & $0.021 \pm 0.007$ & $\mathrm{NA}$ \\
\hline$\omega_{\text {bend }}$ for $\chi_{\text {bend }}^{(2)}$ & $1672.0 \pm 0.9$ & $\mathrm{NA}$ & $1672.0 \pm 0.9$ & $\mathrm{NA}$ & $1672.0 \pm 0.9$ & $\mathrm{NA}$ \\
\hline$A_{\text {bend }}$ for $\chi_{\text {bend }}^{(3)}$ & $-0.541 \pm 0.031$ & $\mathrm{NA}$ & $-0.541 \pm 0.031$ & $\mathrm{NA}$ & $-0.541 \pm 0.031$ & $\mathrm{NA}$ \\
\hline
\end{tabular}

Table S2. The fitting parameters of the complex SFG spectra at water-DPPG interfaces. The error was obtained from the fits.

\begin{tabular}{|c|c|c|c|c|c|c|}
\hline DPPG & $\mathrm{H}_{2} \mathrm{O}$ & $\mathrm{D}_{2} \mathrm{O}$ & $\mathrm{H}_{2} \mathrm{O}$ & $\mathrm{D}_{2} \mathrm{O}$ & $\mathrm{H}_{2} \mathrm{O}$ & $\mathrm{D}_{2} \mathrm{O}$ \\
\hline $\mathrm{NaCl}$ & \multicolumn{2}{|c|}{$1 \mathrm{M}$} & \multicolumn{2}{|c|}{$10 \mathrm{mM}$} & \multicolumn{2}{|c|}{$0.1 \mathrm{mM}$} \\
\hline$A_{\mathrm{NR}}$ & $0.011 \pm 0.001$ & $0.009 \pm 0.001$ & $0.004 \pm 0.001$ & $0.003 \pm 0.001$ & $0.004 \pm 0.001$ & $0.005 \pm 0.001$ \\
\hline$\phi_{\mathrm{NR}}(\mathrm{rad})$ & $3.02 \pm 0.01$ & $3.10 \pm 0.01$ & $3.03 \pm 0.03$ & $3.16 \pm 0.04$ & $1.81 \pm 0.04$ & $2.52 \pm 0.03$ \\
\hline$A_{\mathrm{C}=\mathrm{O}, 1}$ & $-0.24 \pm 0.01$ & $-0.27 \pm 0.01$ & $-0.40 \pm 0.05$ & $-0.36 \pm 0.05$ & $-0.28 \pm 0.02$ & $-0.28 \pm 0.02$ \\
\hline$\omega_{\mathrm{C}=\mathrm{O}, 1}\left(\mathrm{~cm}^{-1}\right)$ & \multicolumn{2}{|c|}{$1718.9 \pm 0.4$} & \multicolumn{2}{|c|}{$1723.4 \pm 1.4$} & \multicolumn{2}{|c|}{$1717.7 \pm 0.8$} \\
\hline$\Gamma_{\mathrm{C}=\mathrm{O}, 1}\left(\mathrm{~cm}^{-1}\right)$ & \multicolumn{2}{|c|}{$12.0 \pm 0.6$} & \multicolumn{2}{|c|}{$19.3 \pm 1.1$} & \multicolumn{2}{|c|}{$14.2 \pm 0.8$} \\
\hline$A_{\mathrm{C}=\mathrm{O}, 2}$ & $0.11 \pm 0.01$ & $0.12 \pm 0.01$ & $0.22 \pm 0.04$ & $0.23 \pm 0.04$ & $0.20 \pm 0.02$ & $0.21 \pm 0.02$ \\
\hline$\omega_{\mathrm{C}=\mathrm{O}, 2}\left(\mathrm{~cm}^{-1}\right)$ & \multicolumn{2}{|c|}{$1741.6 \pm 0.7$} & \multicolumn{2}{|c|}{$1742.9 \pm 0.7$} & \multicolumn{2}{|c|}{$1738.9 \pm 0.6$} \\
\hline$\Gamma_{\mathrm{C}=\mathrm{O}, 2}\left(\mathrm{~cm}^{-1}\right)$ & \multicolumn{2}{|c|}{$10.7 \pm 1.0$} & \multicolumn{2}{|c|}{$12.7 \pm 1.1$} & \multicolumn{2}{|c|}{$12.0 \pm 0.8$} \\
\hline$A_{\text {bend }}$ for $\chi_{\text {bend }}^{(2)}$ & $-0.130 \pm 0.007$ & NA & $-0.130 \pm 0.007$ & NA & $-0.130 \pm 0.007$ & NA \\
\hline$\omega_{\text {bend }}$ for $\chi_{\text {bend }}^{(2)}$ & $1652.4 \pm 1.1$ & NA & $1652.4 \pm 1.1$ & NA & $1652.4 \pm 1.1$ & NA \\
\hline$A_{\text {bend }}$ for $\chi_{\text {bend }}^{(3)}$ & $-0.541 \pm 0.031$ & NA & $-0.541 \pm 0.031$ & NA & $-0.541 \pm 0.031$ & NA \\
\hline
\end{tabular}



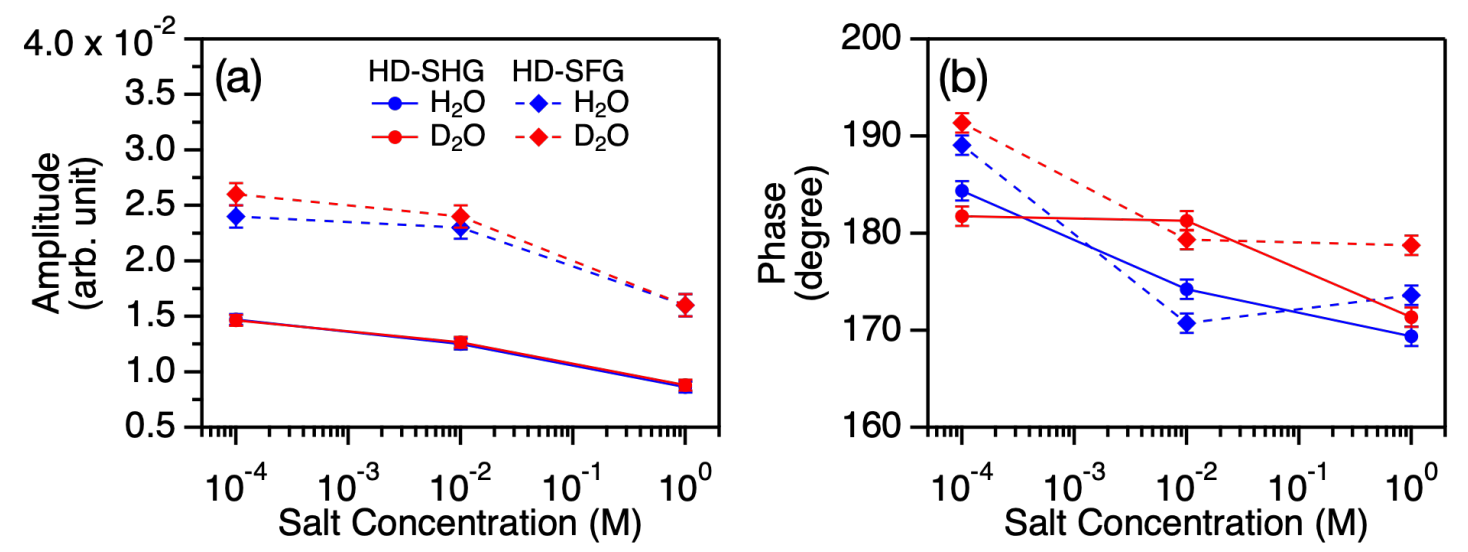

Figure S5. (a, b) Amplitude (a) and phase (b) of the non-resonant contribution obtained by HD-SHG as well as the fit to the HD-SFG spectra for the water-DPTAP interface (Table $\mathrm{S} 1)$. The error bars indicate the $95 \%$ confidence intervals. The $10 \mathrm{mM}$ data are displayed in Figure S3 in the Supporting Information.

\section{Phase Accuracy of the collinear HD-SFG measurement}

To evaluate the phase accuracy of our HD-SFG measurement, we measured $\mathrm{D}_{2} \mathrm{O}$-air interface sample without lipid monolayer. Figure S6 shows the real part and the imaginary part spectra of the measured $\chi_{\mathrm{eff}}^{(2)}(\omega)$. Because there is no specific vibrational mode at $\mathrm{D}_{2} \mathrm{O}$ air interface, the spectral region of interest is dominated only by the non-resonant contribution, displaying zero contribution in the imaginary part. As such, we conclude that no phase calibration is needed. 


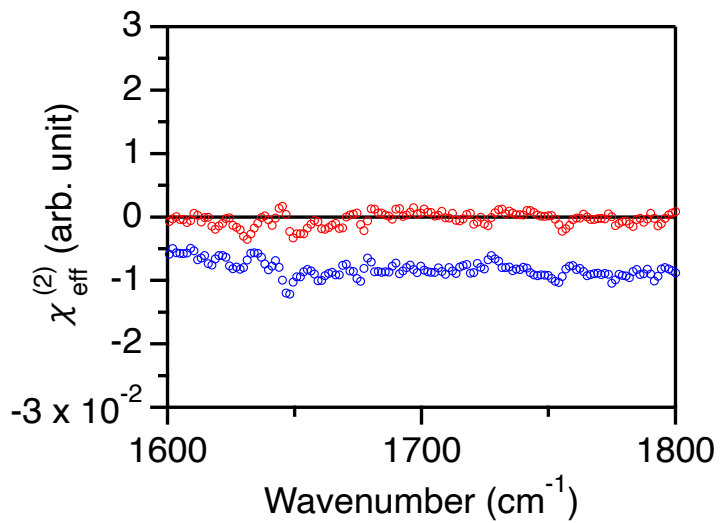

Figure S6. HD-SFG spectra at the $\mathrm{D}_{2} \mathrm{O}$-air interface. The blue and red data points indicate the real and imaginary part, respectively.

\section{REFERENCES}

(1) Sung, W.; Seok, S.; Kim, D.; Tian, C. S.; Shen, Y. R. Sum-Frequency Spectroscopic Study of Langmuir Monolayers of Lipids Having Oppositely Charged Headgroups. Langmuir 2010, 26, 18266-18272.

(2) Liu, W.; Wang, Z.; Fu, L.; Leblanc, R. M.; Yan, E. C. Y. Lipid Compositions Modulate Fluidity and Stability of Bilayers: Characterization by Surface Pressure and Sum Frequency Generation Spectroscopy. Langmuir 2013, 29, 15022-15031.

(3) Backus, E. H. G.; Bonn, D.; Cantin, S.; Roke, S.; Bonn, M. Laser-Heating-Induced Displacement of Surfactants on the Water Surface. J. Phys. Chem. B 2012, 116, 2703-2712.

(4) Nihonyanagi, S.; Mondal, J. A.; Yamaguchi, S.; Tahara, T. Structure and Dynamics of Interfacial Water Studied by Heterodyne-Detected Vibrational SumFrequency Generation. Annu. Rev. Phys. Chem. 2013, 64, 579-603.

(5) Seki, T.; Sun, S.; Zhong, K.; Yu, C.; Machel, K.; Dreier, L. B.; Backus, E. H. G.; Bonn, M.; Nagata, Y. Unveiling Heterogeneity of Interfacial Water through the Water Bending Mode. J. Phys. Chem. Lett. 2019, 10, 6936-6941. 
(6) Ahmed, M.; Nihonyanagi, S.; Kundu, A.; Yamaguchi, S.; Tahara, T. Resolving the Controversy over Dipole versus Quadrupole Mechanism of Bend Vibration of Water in Vibrational Sum Frequency Generation Spectra. J. Phys. Chem. Lett. 2020, 9123-9130.

(7) Seki, T.; Sun, S.; Zhong, K.; Yu, C. C.; MacHel, K.; Dreier, L. B.; Backus, E. H. G.; Bonn, M.; Nagata, Y. SI- Unveiling Heterogeneity of Interfacial Water through the Water Bending Mode. J. Phys. Chem. Lett. 2019.

(8) Dreier, L. B.; Bonn, M.; Backus, E. H. G. Hydration and Orientation of Carbonyl Groups in Oppositely Charged Lipid Monolayers on Water. J. Phys. Chem. B 2019, 123, 1085-1089.

(9) Bard, A. J.; Faulkner, L. R. Electrochemical Methods: Fundamentals and Applications; wiley New York, 2001; Vol. 2. 